CASE REPORT

\title{
Fibroplasia Ossificans Progressiva: A Case Report of a Rare Disease Entity
}

\author{
Daniel Solomon $^{1 *}$, Iyasu Wakjira ${ }^{1}$, Daniel Hailu ${ }^{2}$, Yocabel Gorfy ${ }^{2}$
}

\section{OPEN ACCESS}

Citation: Daniel Solomon, Iyasu Wakjira, Daniel Hailu, Yocabel Gorfy. Fibroplasia Ossificans Progressiva: A Case Report of a Rare Disease Entity. Ethiop J Health Sci. 2017;28(4):517.

doi:http://dx.doi.org/10.4314/ejhs.v28i4.17

Received: August 19, 2017

Accepted: August 22, 2017

Published: July 1, 2018

Copyright: (C) 2018 Daniel Solomon., et al. This is an open access article distributed under the terms of the Creative Commons Attribution License, which permits unrestricted use, distribution, and reproduction in any medium, provided the original author and source are credited.

Funding: Nil

Competing Interests: The authors declare that this manuscript was approved by all authors in its form and that no competing interest exists.

\footnotetext{
Affiliation and Correspondence:

${ }^{1}$ Department of Radiology, School of

Medicine, College of Health Sciences,

Addis Ababa University, Ethiopia

${ }^{2}$ Department of Pediatrics and Child Health, School of Medicine, College of Health Sciences, Addis Ababa University, Ethiopia

*Email:dzewdnehsolomon@yahoo.co
}

ABSTRACT

BACKGROUND: Fibrodysplasia ossificans progressiva (FOP), also known as Myositis ossificans progressiva or Munchmeyer's disease, is an extremely rare and disabling genetic condition of congenital skeletal malformations and progressive heterotopic ossification (HO). The disease is characterized by congenital skeletal anomalies and progressive ectopic bone formation in connective tissues such as ligaments, muscles and tendons. The disease has an incidence of about 1 in 2 million population.

CASE DETAILS: We report a case of a 2-year and 8-month old male child with an initial diagnosis of soft tissue sarcoma based on fine needle aspiration (FNAC) of neck swelling. CONCLUSION: Fibroplasia ossificans progressive (FOP) characteristically manifests with bilateral malformation of the great toe and progressive heterotopic ossification (HO). Clinicians and radiologists should be aware of these to prevent permanent disability.

KEYWORDS: Fibroplasia ossificans progressive, myositis ossificans

\section{INTRODUCTION}

Fibrodys plasia Ossificans Progressiva (FOP) or Myositis Ossificans Progressiva is an extremely rare genetic disorder transmitted as an autosomal dominant trait but can occur sporadically (1). It was first described by Guy Patin in 1648, but Munchmeyer reported the first case series in 1869, retaining the eponym Munchmeyer's disease (2). It is characterized by heterotopic ossifications (HO) of connective tissue and skeletal malformations. It has a prevalence of $1 / 2,000,000$ and is not specific to race, habitat or gender (4). Skeletal abnormalities include monophalangeic great toes with hallux valgus, short first metacarpals, microdactyly, cleinodactyly of the little finger, and abnormalities of the spine. HO characteristically involves the muscles of the head, neck and back in early childhood followed by the shoulders and arms. The muscles around the hips are involved later.

DOI: http://dx.doi.org/10.4314/ejhs.v28i4.17 


\section{CASE REPORT}

Imaging Findings: We report a 2- year and 8 month old male child from our Pediatric Oncology Department with a diagnosis of soft tissue sarcoma based on fine needle aspiration (FNAC) of neck swelling. The patient had a history of minor fall 6 months back. Skull radiographs and head and neck CT and MR showed only soft tissue swelling on the forehead. Two months after the accident, he presented with swelling of the neck and scalp of one-week duration which on imaging showed non-specific multifocal soft tissues swellings and dermatomyositis, respectively. No $\mathrm{HO}$ was noted. FNAC from the neck swelling suggested a soft tissue sarcoma. Swellings spontaneously subsided after four weeks. However, on follow-up, it re-appeared on the back of the neck and disappeared without treatment. Six months after initial presentation, he developed neck stiffness and hard lesions on the nape and the arms. Physical examination showed multiple hard masses on the nape, back and bilateral arms. There was hallux valgus of both great toes.

Further imaging showed multiple extraskeletal ossifications and hallux valgus of both great toes. Routine laboratory investigations and serum calcium were normal. Within two weeks, after steroid therapy, he showed marked improvement, and could walk unsupported, achieving full range flexion and mild extension of the neck. Swellings on the scalp and right knee from minor traumas in the succeeding months responded to steroids; however, he was unable to abduct his arms. He had developed hypertension following prolonged use of steroids. He was on regular follow-up.

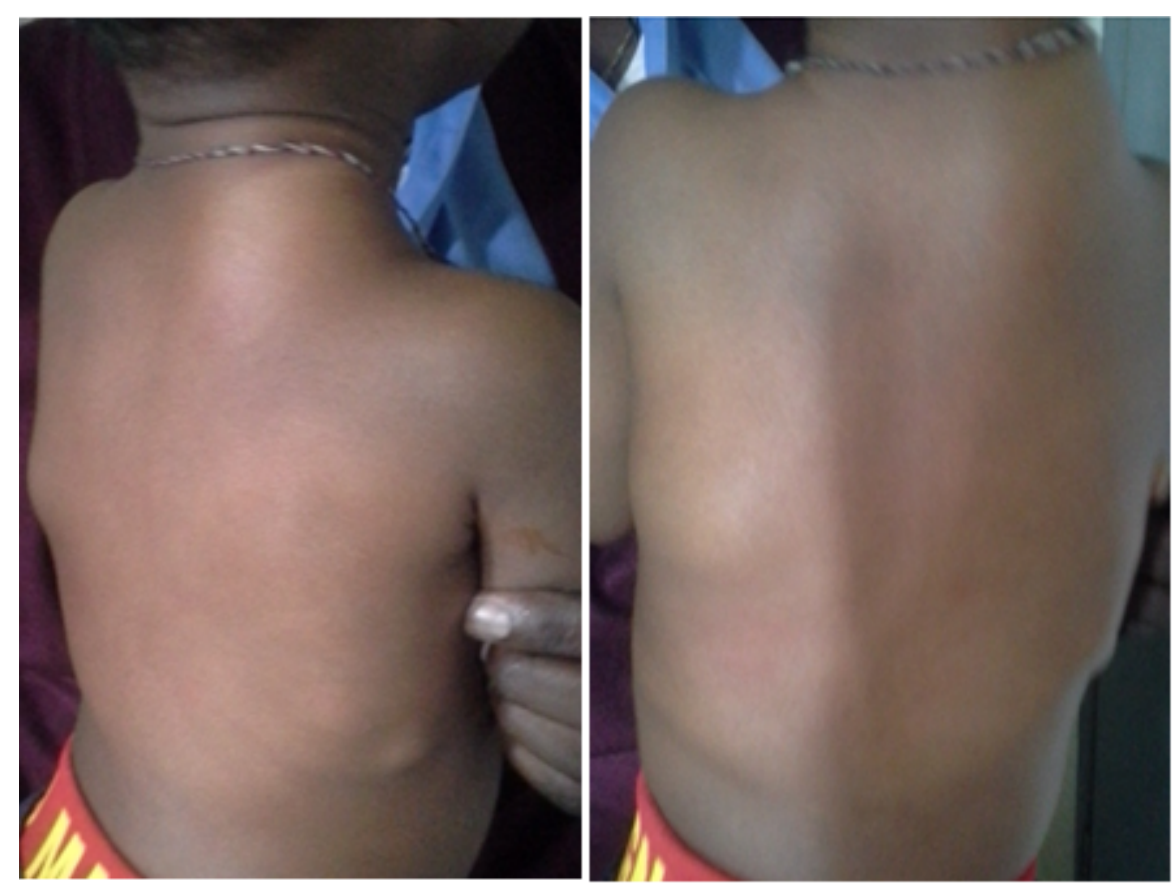

Figure 1: Clinical photograph showing multiple hard swellings over in the nape, below the left scapular, and paravertebral region 

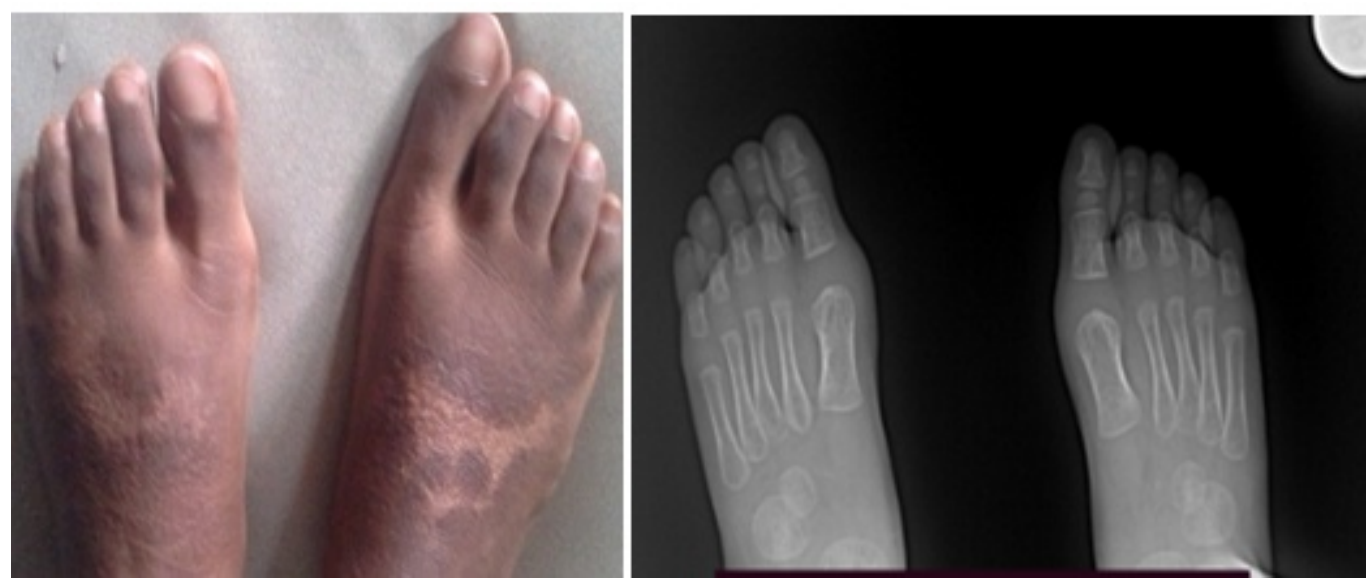

Figure 2: Clinical photograph and frontal radiograph of the feet shows bilateral hallux valgus malformation

\section{DISCUSSION}

Transmission is autosomal dominant but can occur sporadically with a wide range of expressivity and penetrance. Recent gene mappings studies related the FOP gene to chromosome 17q21-22 while others localized it to 4q27-31. The pathogenesis of ectopic bone formation is yet to be described, but overproduction of bone-morphogenetic protein-4 (BMP-4) seen in lesion cells and lymphocytic cells has been reported as one of the factors (1).

Symptoms appear in the first decade of life, and patients appear normal at birth except for the characteristic malformations of the great toes found in all classically affected individuals. The average age of onset of ectopic ossification is 5 years $(1,5)$. In classic FOP, two clinical or radiological features are seen; namely, bilateral malformation of the great toes and progressive $\mathrm{HO}$ in specific anatomic patterns, typically occurring first in the dorsal, axial, cranial, and proximal areas of the body and later appendicular, caudal, and distal areas. However, the diaphragm, tongue, extra ocular muscles, cardiac and smooth, are unexplainably spared $(1,5)$.

Clinically, intermittent symptoms of heat, edema, and painful mass, with erratic remissions and exacerbations occur, resulting in heterotopic bone formation through an endochondral process in muscles, tendons, ligaments, fascia and aponeuroses. Permanent immobility may result as skeletal muscles and connective tissues are replaced by ribbons, sheets and plates of heterotopic bone. Surgery, including biopsy, aggravates the process of the disease. Flare-ups can be induced with influenza-like illness or trivial trauma such as intramuscular injections or minor bumps. Plain films, scintigraphy, CT and MRI can be used to evaluate FOP.

Except fordeformity of the great toes and soft tissue swelling, plain radiographs appear normal early in the course of this illness, but CT is highly sensitive in detecting calcifications and early preosseous lesions with precise anatomic location. However, HO with bony bridging between the axial and appendicular skeleton are seen in advanced cases $(4,5)$. Pre-osseous lesions appear as swelling and edema of the muscular fascial planes and muscular bundles. Hagiwara showed MRI features of pre-osseous FOP as low signal intensity on T1 and high signal intensity on T2weighted images with marked contrast enhancement owing to high vascularity in fibroproliferative tissues. Distinctive pattern of spread along the fascial planes as sheet-like mass between muscles are diagnostic on MRI in early pre-osseous stage $(1,4)$.

The process of lesion formation has defined stages of histological progression. The earliest stage (1A) shows extensive perivascular lymphocytic infiltration. Stage 1B has lymphocytic migration into muscle. Stage $1 \mathrm{C}$ initiates fibroproliferative reaction, with invasion into and replacement of muscle tissue. In stage $2 \mathrm{~A}$, fibroproliferation with intense vascularity occurs while

DOI: http://dx.doi.org/10.4314/ejhs.v28i4.17 
in stage $2 \mathrm{~B}$, cartilage formation is initiated, and in the final stage (2C), frank enchondral bone formation occurs.

At the end of this process, the lesions show histologically mature lamellar bone and marrow elements and macroscopic features of modeling and remodeling similar to normo-tropic skeleton. Therapy aims at prevention of flare-ups and is mostlysupportive. A four-day course of high-dose corticosteroids preferably within the first 24 hours of a flare-up may help limit the intense lymphocytic infiltration and tissue edema in the early stages. For submandibular swelling, prolongation of duration of therapy with tapering, until swelling resolves, is recommended because of increased risk of deterioration. Although efficacy of mast-cell inhibitors is presently unknown, the presence of abundant mast cells in all histological stages of FOP suggest use of mastcell stabilizing, long-acting, non-sedating antihistamines, i.e. leukotriene inhibitors, nonsteroidal anti-inflammatory medications and COX-2 inhibitors may limit progression of early disease. Bone mineralization inhibitor Etidronate, an amino bisphosphonate, has been used because of its potential to impair ossification at high dose, but may cause osteomalacia and impair ossification of normal bone.

Differential diagnosis includes causes of metastatic calcifications (e.g., idiopathic calcinosis universalis, dermatomyositis, idiopathic tumoral calcinosis and disorders of calcium metabolism). In the case of the above conditions and in contrast to FOP, the dense calcific lesions remain without maturing into trabecular bone.

- Differentiation of infantile desmoid-type fibromatosis from pre-osseous lesions of FOP is important because of similarity in age and pathology. On MRI, FOP shows homogenous enhancement and spreads along the fascial planes as sheet-like mass between muscles while infantile desmoids-type fibromatosis shows heterogeneous signal and is poorly marginated (1).
In summary, FOP characteristically demonstrates bilateral malformation of the great toes. In addition to this finding, there is progressive HO. Pediatricians and radiologists should be wary of these typical findings to establish the diagnosis and prevent permanent disability.

\section{ACKNOWLEDGMENT}

We would like to express our sincere gratitude to the parents of the patient. We would also like to thank the staff in the Departments of Radiology and Pediatric Oncology for their support.

\section{REFERENCES}

1. Hiroaki Hagiwara, Noriko Aida, Jiro Machid, Kazutoshi Fujita, Shigeharu Okuzumi, Gen Nishimura Contrast-Enhanced MRI of an Early Preosseous Lesion of Fibrodysplasia Ossificans Progressivain a 21-Month-Old Boy. AJR, 2003;181:1145-1147.

2. Illingworth RS. Myositis Ossificans Progressiva (Munchmeyer's Disease) Brief review with report of two cases treated with corticosteroids and observed for 16 years. Archives of Disease in Childhood, 1971, 46, 264.

3. Zafar A, Maroof SA, Ali Z. A case of fibrodysplasia ossificans progressiva with primary amenorrhea. Kust Med J, 2010; 2(2): 73-75.

4. Nadia Shaikh, FehminaArif: Fibrodysplasia Ossificans Progressiva: Journal of Pakistan Medical Association (JPMA); 2011: 61 (4): 397-399.

5. Ishaq Al-Salmi, Sameer Raniga, and Aymen AlHadidi. Fibrodysplasia Ossificans Progressiva- Radiological Findings: A Case Report. Oman Medical Journal, 2014; 29(5):368-370. 